\title{
NMR assignments of the FKBP-type PPIase domain of FKBP42 from Arabidopsis thaliana
}

\author{
Noelia Inés Burgardt • Miriam Linnert • \\ Matthias Weiwad • Markus Geisler • \\ Christian Lücke
}

\begin{abstract}
The Atfkbp42 gene is associated with reduced and disoriented growth of Arabidopsis thaliana. Resonance assignments are reported for the FKBP-type PPIase domain of $A t \mathrm{FKBP} 42$. Signal intensities reveal an additional structure element that is atypical for such FKBP domains.
\end{abstract}

Keywords FK506-binding protein - Twisted dwarf 1 . Auxin transport $\cdot$ Signal transduction $\cdot$ Trp-Pro stacking

\section{Biological context}

Arabidopsis thaliana FKBP42 (AtFKBP42), also referred to as twisted dwarf 1 (twd1), belongs to the enzyme class of peptidyl-prolyl cis/trans isomerases (PPIases). Twd1 deletion mutants are reduced in height and exhibit disoriented growth of all organs, but develop fertile flowers and seeds (Geisler et al. 2003). The AtFKBP42 protein comprises a single FKBP-type PPIase domain, a tripartite tetratricopeptide repeat (TPR) motif, a putative calmodulin binding site and a hydrophobic C-terminal membrane

N. I. Burgardt · M. Linnert · M. Weiwad · C. Lücke $(\bowtie)$

Max Planck Research Unit for Enzymology of Protein Folding, Weinbergweg 22, 06120 Halle (Saale), Germany

e-mail: luecke@enzyme-halle.mpg.de

\section{N. I. Burgardt}

School of Pharmacy and Biochemistry, Institute of Biochemistry and Biophysics (IQUIFIB), University of Buenos Aires, Junín 956, C1113AAD Buenos Aires, Argentina

\section{Geisler}

Department of Plant Biology, University of Fribourg,

Chemin du Musée 10, 1700 Fribourg, Switzerland anchor that localizes the protein both on the central vacuole and on the plasma membrane (Geisler et al. 2003; Kamphausen et al. 2002). The FKBP domain of AtFKBP42, however, does not exhibit PPIase activity towards standard peptide substrates. Moreover, the domain exhibits no affinity for the immunosuppressive drug FK506 (Kamphausen et al. 2002). The TPR domain of the protein interacts with AtHSP90, comparable to the binding of the human multidomain proteins FKBP38, FKBP51 and FKBP52 to HSP90 in mammalian cells (Kamphausen et al. 2002). Several studies have highlighted the importance of the HSP90 chaperone in plant development and responsiveness to external stimuli (Sangster and Queitsch 2005).

The FKBP domain of AtFKBP42 physically interacts with the second nucleotide-binding domain (NBD2) in the cytoplasmic C-terminal domain of the plasma membranelocalized ATP-binding cassette (ABC) transporters AtPGP1 and AtPGP19 (Geisler et al. 2003). Both P-glycoproteins have been shown to directly mediate cellular auxin efflux (Geisler et al. 2005; Lin and Wang 2005). The plant hormone auxin directly influences cell growth, but also controls numerous and diverse aspects of plant development (Teale et al. 2006). Atfkbp42 and atpgp1/ atpgp19 deletion mutants both exhibit remarkably overlapping phenotypes, suggesting a regulatory role of AtFKBP42 in PGP-mediated export of auxin (Bouchard et al. 2006; Geisler et al. 2003). The TPR domain of AtFKBP42 forms complexes with multidrug-resistance associated proteins MRP1 and MRP2, two members of another subfamily of $\mathrm{ABC}$ transporters (Geisler et al. 2004). Both MRP transporters localize to the tonoplast and have been shown to be involved in detoxification of organic xenobiotics and herbicides as well as chlorophyll catabolites (Liu et al. 2001; Lu et al. 1998; Tommasini et al. 1998). 


\section{Methods and experiments}

Protein preparation

Isotope-labelled AtFKBP42 $2^{1-180}$ was prepared as previously described (Kamphausen et al. 2002), except that the BL21 CodonPlus RIL cells (Stratagene, LJ, USA) were grown at $30^{\circ} \mathrm{C}$ in $\mathrm{M} 9$ minimal medium and harvested $3 \mathrm{~h}$ after induction with $0.5 \mathrm{mM}$ isopropyl- $\beta$-D-thiogalactopyranoside (IPTG). The NMR sample contained $2 \mathrm{mM}$ ${ }^{13} \mathrm{C} /{ }^{15} \mathrm{~N}$-labelled FKBP42 $2^{1-180}$ in $10 \mathrm{mM} \mathrm{Na}{ }_{2} \mathrm{HPO}_{4}$ buffer $\left(5 \% \mathrm{D}_{2} \mathrm{O}, \mathrm{pH} 7.0\right)$.

NMR spectroscopy

All NMR spectra were acquired at $25^{\circ} \mathrm{C}$ using Bruker DRX 500 and Avance 800 (with cryoprobe) spectrometers, with the carrier placed in the center of the spectrum on the water resonance which was suppressed by applying a WATERGATE sequence. Quadrature detection in the indirectlydetected dimension was obtained by the States-TPPI method. All NMR spectra were acquired and processed on Silicon Graphics computers using the program XWINNMR 3.5 (Bruker Bio-Spin, Rheinstetten, Germany). A $90^{\circ}$ phase-shifted squared sine-bell function was used for apodization in all dimensions. Polynomial baseline correction was applied to the processed spectra in the directly-detected ${ }^{1} \mathrm{H}$ dimension. The chemical shifts were referenced to external DSS in order to ensure consistency among all spectra (Wishart et al. 1995). Spectra were analyzed with SPARKY 3 (University of California SF, USA).

In addition to $2 \mathrm{D}{ }^{1} \mathrm{H} /{ }^{15} \mathrm{~N}$-TROSY, ${ }^{1} \mathrm{H} /{ }^{15} \mathrm{~N}-\mathrm{HSQC}$ and ${ }^{1} \mathrm{H} /{ }^{13} \mathrm{C}$-HSQC spectra, the following 3D triple-resonance datasets were collected. The sequential backbone assignment is based on HNCA and HNCACB experiments. Sidechain resonances were derived from $\mathrm{CC}(\mathrm{CO}) \mathrm{NH}$ and $\mathrm{H}(\mathrm{CC})(\mathrm{CO}) \mathrm{NH}$ experiments (17.5 ms spinlock time each). Carbonyl resonances were determined from $\mathrm{HNCO}$ and $\mathrm{HN}(\mathrm{CA}) \mathrm{CO}$ spectra.

\section{Assignments and data deposition}

Resonance assignments were obtained for nearly all amide protons and backbone heavy atoms as well as $89 \%$ of the aliphatic side-chain ${ }^{13} \mathrm{C}$ resonances. Missing are resonances belonging to Met1, since the backbone amide group of Asp2 could not be detected, and of Pro37 and Pro144, each of which commences a diproline segment. The resonance assignment of At $\mathrm{FKBP} 42^{1-180}$ has been deposited at the BioMagResBank (http://www.bmrb.wisc.edu) database under accession number BMRB-17998.

Figure 1a shows the AtFKBP42 $2^{1-180}$ backbone amide resonance assignments. The assignment procedure was complicated by extended non-structured segments at the $\mathrm{N}$ - and C-terminal ends of the $A t \mathrm{FKBP} 42^{1-180}$ construct,
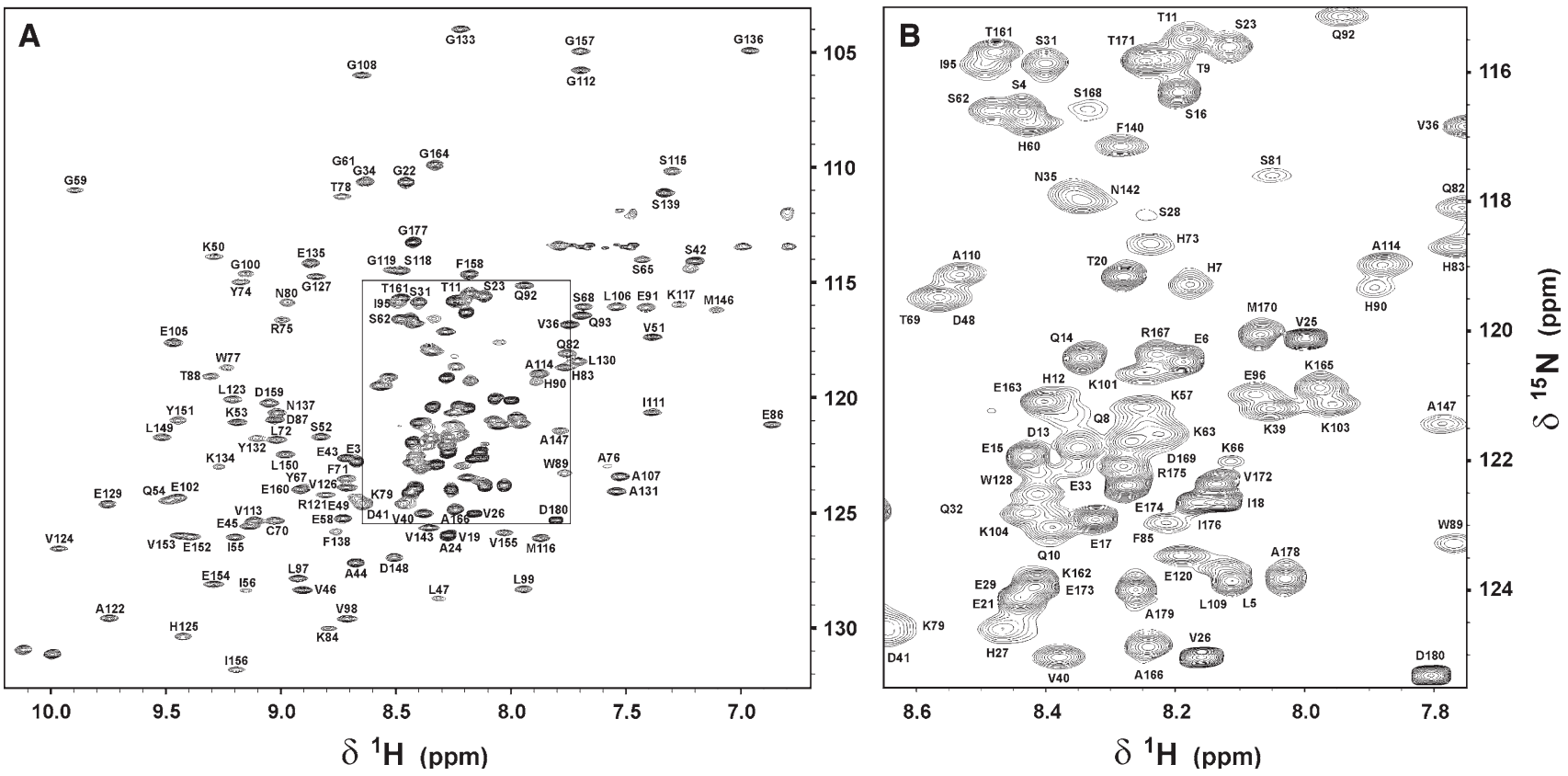

Fig. $1{ }^{1} \mathrm{H} /{ }^{15} \mathrm{~N}$-TROSY spectrum showing the backbone amide resonance assignments of $A t \mathrm{FKBP} 42^{1-180}$ (a); close-up of the central section featuring most of the high intensity peaks (b) 
which featured high intensity peaks in the central part of the ${ }^{1} \mathrm{H} /{ }^{15} \mathrm{~N}$-TROSY spectrum (Fig. 1b).

Chemical shift index (CSI) analysis (Wishart and Sykes 1994) of AtFKBP42 $2^{1-180}$ revealed a secondary structure pattern in the FKBP domain (Fig. 2) analogous to the corresponding crystal structure (Weiergräber et al. 2006). A short $\beta$-strand segment (Lys84-Asp87) belonging to the FKBP domain was not identified by the CSI consensus; but a hitherto unknown $\beta$-strand element (Ile18-Thr20) was found near the N-terminal end of AtFKBP42 ${ }^{1-180}$.

High intensity peaks were observed for segments Met1 to Glu29 and Val172 to Asp180 at the N- and C-terminal ends, respectively. This is in agreement with previous X-ray data (Weiergräber et al. 2006), where segments

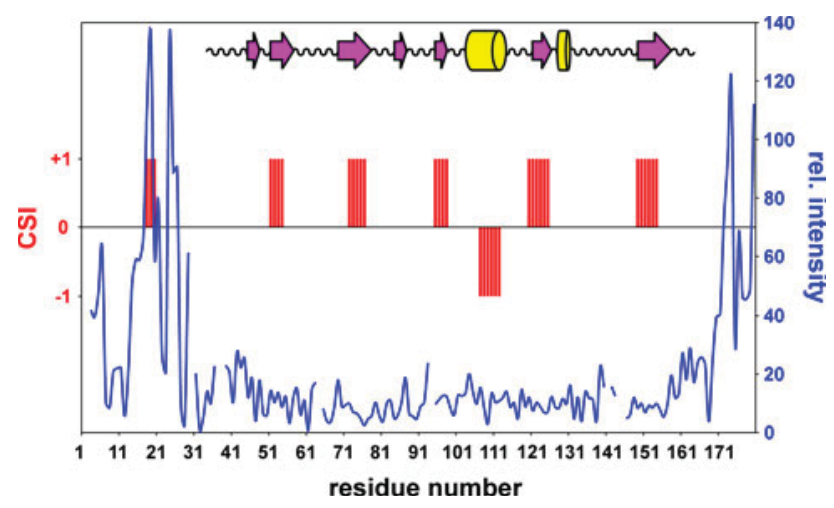

Fig. 2 Alignment of CSI consensus values (red bars) and relative signal intensities (blue line) with the secondary structure elements (cartoon) of the AtFKBP42 crystal structure (PDB ID code $2 \mathrm{~F} 4 \mathrm{E}$ ). Positive and negative CSI bars indicate $\beta$-strand and $\alpha$-helix segments, respectively. In the secondary structure cartoon at the top, arrows (magenta) and cylinders (yellow) represent $\beta$-strands and $\alpha$-helices, respectively

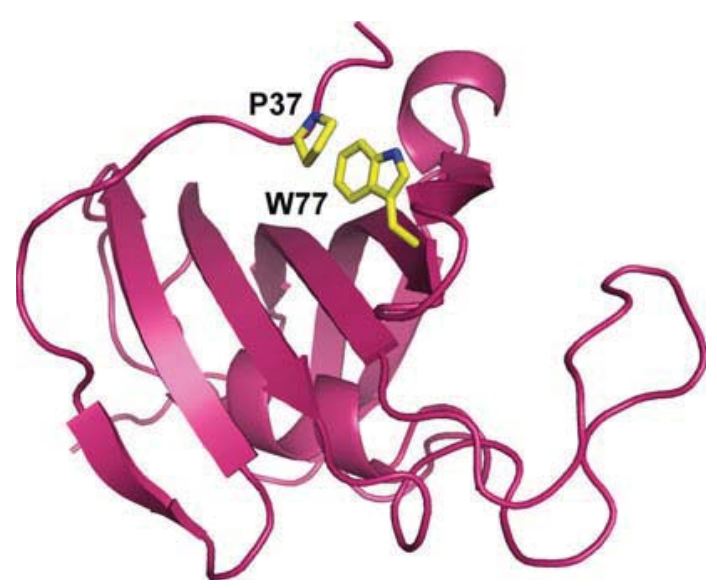

Fig. 3 Cartoon representation of the FKBP domain of AtFKBP42 (PDB ID code 2F4E). The N-terminal segment Gly34 to Ala44 wraps around the convex face of the half $\beta$-barrel. This FKBP-atypical structure element apparently results from a stacking interaction between Pro37 and Trp77 (yellow sticks)
Met1-Glu33 and Lys165-Asp180 of AtFKBP42 ${ }^{1-180}$ showed no traceable electron density presumably due to a high structural disorder. Interestingly, however, residues Gly34 to Ala44, which in the crystal structure are welldefined and wrap around the convex face of the half $\beta$-barrel defining the FKBP domain, feature NMR signal intensities similar to the domain. Hence, also in solution this particular segment (residues Ser31 to Ala44) appears not highly mobile but affixed to the FKBP domain of AtFKBP42, possibly representing an additional FKBPatypical structural feature. Most likely, this clamp around the back of the FKBP domain is held in place by a stacking interaction $(<4 \AA)$ between the rings of Trp77 and Pro37 (Fig. 3).

Acknowledgments The authors are grateful to Prof. Jochen Balbach (University of Halle, Germany) for providing access to the $800 \mathrm{MHz}$ NMR spectrometer. N.I.B. was beneficiary of the Partial Funding Program for Short Stays Abroad of CONICET (Argentina). This work was supported by German Ministry for Research and Education grants BMBF 0315638B and 03IS2211H.

\section{References}

Bouchard R, Bailly A, Blakeslee JJ, Oehring SC, Vincenzetti V, Lee OR, Paponov I, Palme K, Mancuso S, Murphy AS, Schulz B, Geisler M (2006) Immunophilin-like TWISTED DWARF1 modulates auxin efflux activities of Arabidopsis P-glycoproteins. J Biol Chem 281:30603-30612

Geisler M, Kolukisaoglu HU, Bouchard R, Billion K, Berger J, Saal B, Frangne N, Koncz-Kálmán Z, Koncz C, Dudler R, Blakeslee JJ, Murphy AS, Martinoia E, Schulz B (2003) TWISTED DWARF1, a unique plasma membrane-anchored immunophilinlike protein, interacts with Arabidopsis multidrug resistance-like transporters AtPGP1 and AtPGP19. Mol Biol Cell 14:4238-4249

Geisler M, Girin M, Brandt S, Vincenzetti V, Plaza S, Paris N, Kobae Y, Maeshima M, Billion K, Kolukisaoglu UH, Schulz B, Martinoia E (2004) Arabidopsis immunophilin-like TWD1 functionally interacts with vacuolar $\mathrm{ABC}$ transporters. Mol Biol Cell 15:3393-3405

Geisler M, Blakeslee JJ, Bouchard R, Lee OR, Vincenzetti V, Bandyopadhyay A, Titapiwatanakun B, Peer WA, Bailly A, Richards EL, Ejendal KF, Smith AP, Baroux C, Grossniklaus U, Müller A, Hrycyna CA, Dudler R, Murphy AS, Martinoia E (2005) Cellular efflux of auxin catalyzed by the Arabidopsis MDR/PGP transporter AtPGP1. Plant J 44:179-194

Kamphausen T, Fanghänel J, Neumann D, Schulz B, Rahfeld JU (2002) Characterization of Arabidopsis thaliana AtFKBP42 that is membrane-bound and interacts with Hsp90. Plant J 32: 263-276

Lin R, Wang H (2005) Two homologous ATP-binding cassette transporter proteins, AtMDR1 and AtPGP1, regulate Arabidopsis photomorphogenesis and root development by mediating polar auxin transport. Plant Physiol 138:949-964

Liu G, Sanchez-Fernandez R, Li ZS, Rea PA (2001) Enhanced multispecificity of Arabidopsis vacuolar multidrug resistanceassociated protein-type ATP-binding cassette transporter AtMRP2. J Biol Chem 276:8648-8656

Lu YP, Li ZS, Drozdowicz YM, Hörtensteiner S, Martinoia E, Rea PA (1998) AtMRP2, an Arabidopsis ATP binding cassette 
transporter able to transport glutathione S-conjugates and chlorophyll catabolites: functional comparisons with AtMRP1. Plant Cell 10:267-282

Sangster TA, Queitsch C (2005) The HSP90 chaperone complex, an emerging force in plant development and phenotypic plasticity. Curr Opin Plant Biol 8:86-92

Teale WD, Paponov IA, Palme K (2006) Auxin in action: signalling, transport and the control of plant growth and development. Nat Rev Mol Cell Biol 7:847-859

Tommasini R, Vogt E, Fromenteau M, Hörtensteiner S, Matile P, Amrhein N, Martinoia E (1998) An ABC-transporter of
Arabidopsis thaliana has both glutathione-conjugate and chlorophyll catabolite transport activity. Plant J 13:773-780

Weiergräber OH, Eckhoff A, Granzin J (2006) Crystal structure of a plant immunophilin domain involved in regulation of MDR-type ABC transporters. FEBS Lett 580:251-255

Wishart DS, Sykes BD (1994) The ${ }^{13} \mathrm{C}$ chemical-shift index: a simple method for the identification of protein secondary structure using ${ }^{13} \mathrm{C}$ chemical-shift data. J Biomol NMR 4:171-180

Wishart DS, Bigam CG, Yao J, Abildgaard F, Dyson HJ, Oldfield E, Markley JL, Sykes BD (1995) ${ }^{1} \mathrm{H},{ }^{13} \mathrm{C}$ and ${ }^{15} \mathrm{~N}$ chemical shift referencing in biomolecular NMR. J Biomol NMR 6:135-140 